\title{
Quantitative Trait Locus Analysis of Stalk Strength in Four Maize Populations
}

\author{
Sherry A. Flint-Garcia, Chaba Jampatong, Larry L. Darrah,* and Michael D. McMullen
}

\begin{abstract}
Stalk lodging in maize (Zea mays L.) causes yield losses estimated to range from 5 to $20 \%$ annually worldwide. Selection for rind penetrometer resistance (RPR) has proven useful in enhancing germplasm for stalk strength, and therefore improving stalk lodging resistance. We conducted quantitative trait locus (QTL) analysis for RPR in four $F_{2: 3}$ populations. The populations were constructed by means of combinations of MoSCSSS-High (selection for high RPR), MoSCSSSLow (selection for low RPR), MoSQB-Low (selection for low stalk crushing strength), inbred line Mo47, and inbred line B73. Individuals in each population were genotyped for simple sequence repeat (SSR) or restriction fragment length polymorphism (RFLP) markers, and data were collected for RPR over multiple locations and replications. Means combined over environments were used as trait data for composite interval mapping by QTL Cartographer. Eight, 10, eight, and nine single-effect QTL and four, two, zero, and five epistatic interactions were detected for RPR in the four populations. Multilocus models, including the single-effect QTL and epistatic interactions, accounted for $33.4,44.7,48.4$, and $58.7 \%$ of the total phenotypic variation. These data clearly indicate the complex nature of stalk strength. One chromosomal region contained a QTL from all four populations, while two QTL were in common among three of the four populations and five QTL were in common between two populations. Candidate genes that overlap QTL confidence intervals include those involved in lignin synthesis, the phenylpropanoid pathway, and the timing of vegetative phase change.
\end{abstract}

$\mathrm{S}$ TALK LODGING RESISTANCE continues to be an important aspect of plant standability in maize. Stalk lodging is breakage of the stalk at or below the ear, which may result in loss of the ear at harvest. Stalk lodging can be caused by both biotic and abiotic factors including pathogens, insects, and wind. Gibberella zeae (Schwein.) (anamorph: Fusarium graminearum Schwabe), F. moniliforme (Sheld.), and Diplodia zeae (Schwein.) are all fungal pathogens that cause stalk rot, thus reducing the strength of the stalk and increasing susceptibility to lodging (Smith and White, 1988). Insect pests that increase stalk lodging by tunneling in the USA include the European corn borer (ECB) (Ostrinia nubilalis Hübner) and the Southwestern corn borer (Diatraea grandiosella Dyar) (Mihm, 1985). The increased use of reduced tillage has likely contributed to increased incidence of stalk

S.A. Flint-Garcia, Genetics Department, North Carolina State University, Gardner Hall, Raleigh, NC 27695; C. Jampatong, National Corn and Sorghum Research Center, Kasetsart Univ., Klangdong, Pakchong, Nakhonratchasima 30320, Thailand; L.L. Darrah and M.D. McMullen, USDA-ARS Plant Genetics Research Unit and Department of Agronomy, University of Missouri-Columbia, Curtis Hall, Columbia, MO 65211. Mention of a trademark or proprietary product does not constitute a guarantee, warranty, or recommendation of the product by the USDA or the University of Missouri, and does not imply its approval to the exclusion of others that may be more suitable. Received 13 Nov. 2001. *Corresponding author (DarrahL@missouri. edu).

Published in Crop Sci. 43:13-22 (2003). rots in maize. High yielding cultivars, increased plant densities, and improvements in soil fertility are generally accepted to have also contributed to increased stalk lodging.

Stalk lodging counts are unreliable as a measure of stalk lodging resistance because the expression of stalk lodging is affected by diseases, insects, and wind. Data summarized from the national white food corn performance trials for the years 1986 to 2000 indicate that visual counts of percentages of stalk lodging have coefficients of variation (CVs) in the range of 22 to $150 \%$ with an average of $82 \%$ (L.L. Darrah, unpublished data). Several methods have been devised to measure stalk strength to improve stalk lodging resistance. Zuber and Grogan (1961) developed a stalk crushing strength (SCS) technique whereby a $5.1-\mathrm{cm}$ dried section of stalk from the second or third internode above the ground was crushed vertically with a hydraulic press. The force required to "pop" the rind was significantly negatively correlated with stalk lodging in multiple studies (Zuber and Grogan, 1961; Thompson, 1963). The CVs for SCS ranged from 8 to $35 \%$. However, because of the destructive nature of sampling by SCS, an alternative method of evaluating stalk strength was desired. More emphasis was placed on rind strength because studies indicated that the rind contributed 50 to $80 \%$ of the stalk strength (Zuber et al., 1980). Sibale et al. (1992) described use of a modified electronic rind penetrometer to measure stalk strength and found a highly significant correlation between SCS and rind penetrometer resistance (RPR) with a CV of $10.5 \%$ for RPR. More importantly, RPR was significantly and negatively correlated with stalk lodging (Chesang-Chumo, 1993; McDevitt, 1999; Spiess, 1995; Jampatong, 1999).

Little is known about the genetic nature of stalk lodging resistance and RPR. Previous studies have investigated the genetics of stalk strength at a broad level and found that multiple genic regions were involved in RPR (Heredia-Diaz et al., 1996; Lee et al., 1996). These studies, however, are too limited to identify the biochemical and physiological pathways underlying in stalk strength or to provide a basis for marker-assisted selection. The present study is first attempt to explore known candidate genes that might be associated with RPR. When

\footnotetext{
Abbreviations: CV, coefficient of variation; ECB, European corn borer; LOD, log-odds ratio; MoSCSSS-High1, MoSCSSS(H24-High Rind Penetrometer)C10S1; MoSCSSS-High2, MoSCSSS(H24-High Rind Penetrometer)C10S2-1; MoSCSSS-High3, MoSCSSS(H24-High Rind Penetrometer)C10S2-2; MoSCSSS-Low, MoSCSSS(H25-Low Rind Penetrometer)C11; MoSQB-Low, MoSQB(S10)C6; PCR, polymerase chain reaction; QTL, quantitative trait locus; $\mathrm{R}^{2}$, percent of phenotypic variation explained; RFLP, restriction fragment length polymorphism; RCBD, randomized complete block; RPR, rind penetrometer resistance; SCS, stalk crushing strength; SSR, simple sequence repeat.
} 
investigating a complex trait such as RPR, where many physiological and anatomical factors are involved, QTL analysis is the most appropriate initial approach to study the underlying genetic mechanisms. The objectives of this study were to (i) locate QTL for rind penetrometer resistance in four maize populations, (ii) estimate their effects, and (iii) identify candidate genes for these QTL.

\section{MATERIALS AND METHODS \\ Population Development}

MoSCSSS is a yellow dent synthetic population formed from 14 inbred lines derived from the Iowa Stiff Stalk synthetic (USDA-ARS and Mo. Agric. Exp. Stn., 1986) (see pedigree abbreviations and population construction in Table 1). Recurrent $\mathrm{S}_{0}$ plant selection for increased and decreased RPR within MoSCSSS yielded MoSCSSS(H24-high rind penetrometer [HRP])C10 and MoSCSSS(H25-low rind penetrometer [LRP]) C11, respectively. MoSQB is a synthetic that was formed from 12 yellow endosperm inbred lines by M. S. Zuber (Gerdes et al., 1993). Recurrent $S_{0}$ plant selection for low SCS within MoSQB yielded MoSQB(S10)C6. To initiate population development without delay, samples of each of MoSCSSS(H24HRP)C10 and MoSQB(S10)C6 were grown in a greenhouse ground bed and evaluated for RPR. We selected the plant with the highest RPR in the MoSCSSS(H24-HRP)C10 sample and the plant with the lowest RPR in the MoSQB(S10)C6 sample. These plants were self pollinated, and the resulting progeny are hereafter referred to as MoSCSSS-High1 and MoSQB-Low, respectively. Because there was no overlap between the two original populations [MoSCSSS(H24-HRP)C10 and MoSQB(S10)C6 ], we believe that adequate parental materials were selected. MoSCSSS-High1 was self pollinated and the derived progeny are referred to as MoSCSSS-High2 and MoSCSSS-High3.

Population 1 was formed by crossing an individual MoSQBLow plant (female) with an individual MoSCSSS-High1 plant. Population 2 was formed by crossing an individual MoSCSSSHigh2 plant (female) with an individual plant from MoSCSSS(H25-LRP) (hereafter referred to as MoSCSSS-Low). Population 3 was formed by crossing Mo47 (female) with an individual MoSCSSS-High3 plant. Population 4 was formed by crossing B73 (female) with Mo47. For all populations, $\mathrm{F}_{1}$ plants were self pollinated yielding $F_{2}$ individuals, which were self pollinated to produce $\mathrm{F}_{2: 3}$ families. Populations 1, 2, 3, and 4 included 282, 291, 291, and $244 \mathrm{~F}_{2: 3}$ families, respectively. The $\mathrm{F}_{2: 3}$ families were sib pollinated to increase seed for phenotypic data collection.

Populations 1 and 2 were designed specifically to map stalk rind strength QTL since both parents were selected for high and low stalk strength phenotypes. Population 4 was initially designed to map QTL for resistance to both first- and second-

Table 1. Pedigrees of germplasm used as parents in Populations $1,2,3$, and 4.

\begin{tabular}{lll}
\hline Population & \multicolumn{1}{c}{ Abbreviation } & \multicolumn{1}{c}{ Pedigree } \\
\hline Population 1 & MoSQB-Low & MoSQB(S10)C6 \\
& MoSCSSS-High1 & MoSCSS(H24-HRP $\dagger$ )C10S1 \\
Population 2 & MoSCSSS-High2 & MoSCSSS(H24-HRP)C10S2-1 \\
& MoSCSSS-Low & MoSCSSS(H25-LRP †)C11 \\
Population 3 & Mo47 & Mo47 \\
& MoSCSSS-High3 & MoSCSSS(H24-HRP)C10S2-2 \\
Population 4 & B73 & B73 \\
& Mo47 & Mo47 \\
\hline
\end{tabular}

$\dagger$ HRP $=$ selection for high rind penetrometer resistance.

† $\mathbf{L R P}=$ selection for low rind penetrometer resistance. generation ECB, with Mo47 as the source of resistance. We decided to evaluate RPR in this population, where neither parent was selected for stalk strength per se. By not maximizing the difference between parents regarding stalk strength, we might be able to identify QTL that were not selected for in the other populations. Because both parents were inbred lines, this type of mapping population and the results it would provide would be more representative of experiments conducted by industry. Population 3 was designed to bridge the gap between Populations 1 and 2 and Population 4. While not the focus of this study, Population 3 would also lays the groundwork for subsequent studies investigating the relationship between stalk strength and stalk tunneling by secondgeneration ECB.

\section{Phenotypic Data Collection}

Locations used for evaluation trials in this study include Hinkson Bottom at Columbia, MO, on Freeburg silt loam; Agronomy Research Center near Columbia, MO, on Mexico silt loam; a site near Grand Pass, MO, in Saline County on Haynie silt loam; a site near Tipton, MO, in Cooper County on Clafork and Crestmeade silt loam; and a site managed by the Illinois Crop Improvement Association near Juana Diaz, Puerto Rico, on San Antón sandy clay loam. All experiments in Missouri were planted as 6.7-m-long single-row plots spaced $0.90 \mathrm{~m}$ apart for a final planting density of 53800 plants ha ${ }^{-1}$. Standard cultural practices were used in fertilization, and weed and pest control for the Missouri experiments. All experiments in Puerto Rico were planted on a raised bed in paired rows with $50.8 \mathrm{~cm}$ between paired rows and $1.32 \mathrm{~m}$ between pairs of rows for a final planting density of 44850 plants ha $^{-1}$. Plots in Puerto Rico were irrigated by means of drip tape positioned between the $50.8-\mathrm{cm}$ rows.

Population 1 was divided into three sets, each containing entries for 94 families and two entries for each of the parents and $F_{1}$. Each set was planted as a $10 \times 10$ triple lattice. Locations included two replications (one replication of three planted was not usable) at the Agronomy Research Center in 1999, and three replications at the Agronomy Research Center and Tipton sites in 2000.

Populations 2 and 3 were divided into three sets apiece, each containing entries for 97 families and one entry of the parents and $F_{1}$. Again, each set was planted as a $10 \times 10$ triple lattice. Locations for both populations included three replications at two locations in Puerto Rico in 1999, and three replications at the Agronomy Research Center and Tipton sites in 2000.

Population 4 was planted as a $16 \times 16$ triple lattice containing 244 families, two entries for each of the parents and $F_{1}$, and six check entries (Jampatong et al., 2002). In 1996, three replications were planted at Grand Pass, and in 1997, three locations were planted with three replications at Grand Pass, Hinkson Bottom, and the Agronomy Research Center.

Rind penetrometer resistance was determined for $10 \mathrm{com}-$ petitive plants plot $^{-1}$ with an electronic rind penetrometer. The rind penetrometer is a modified Accuforce Cadet digital force gage (Ametek, Largo, FL), 22.7-kg capacity, powered by a $9-\mathrm{V}$ alkaline battery (Sibale et al., 1992). About $2 \mathrm{wk}$ after flowering, plants were probed in the middle of the flat side of the internode below the primary ear node. To increase the precision of our RPR measurements, only a single, skilled rind penetrometer operator evaluated a complete set. This allowed for removal of operator effects during subsequent data analysis. 


\section{Molecular Marker Genotype Analysis}

Leaf tissue was collected from $20 \mathrm{~F}_{2: 3}$ plants and bulked to reconstitute the $\mathrm{F}_{2}$ genotype for Populations 1 and 4, or from tissue of $\mathrm{F}_{2}$ plants for Populations 2 and 3. We extracted DNA using either a modified method based on Saghai-Maroof et al. (1984), or by a microextraction method developed at the University of Missouri by D. Davis and G. Xu. Populations 1,2 , and 3 were genotyped with SSR markers while Population 4 was genotyped by RFLP markers.

For genotyping using SSRs, the PCR reaction consisted of $50 \mathrm{ng}$ each SSR primer, $50 \mathrm{ng}$ genomic DNA, and either (i) $1 \times$ PCR Buffer, $2.5 \mathrm{mM} \mathrm{MgCl}, 0.4 \mathrm{~m} M$ of each dNTP, and 0.3 units of Platinum Taq polymerase (Invitrogen, Carlsbad, $\mathrm{CA}$ ) in a final volume of $15 \mu \mathrm{L}$, or (ii) $9.9 \mathrm{~mL}$ of Jumpstart Ready Mix REDTaq PCR reaction mix (Sigma-Aldrich, St. Louis, MO) in a $20-\mu \mathrm{L}$ reaction. The thermocycling program was as follows: $95^{\circ} \mathrm{C}$ for $1 \mathrm{~min}, 65^{\circ} \mathrm{C}$ for $1 \mathrm{~min}$, and $72^{\circ} \mathrm{C}$ (annealing temperature) for $1.5 \mathrm{~min}$ for the first cycle, and then a one-degree decrement for the annealing temperature, each repeated once, until the annealing temperature was $55^{\circ} \mathrm{C}$. The regime thereafter was $95^{\circ} \mathrm{C}$ for $1 \mathrm{~min}, 55^{\circ} \mathrm{C}$ for $1 \mathrm{~min}$, $72^{\circ} \mathrm{C}$ for $1.5 \mathrm{~min}$, repeated for a total of 30 cycles. Amplification products were resolved by electrophoresis on 4 or $5 \%$ $(\mathrm{w} / \mathrm{v})$ super fine resolution-agarose gels (Amresco, Solon, $\mathrm{OH}$ ). Populations 1, 2, and 3 were genotyped with 89, 77, and 86 SSR markers, respectively. For Population 4, genomic DNA was digested with one of six restriction enzymes and transferred to nylon membranes as previously described (Jampatong et al., 2002). Ninety-seven radioactively labeled probes were hybridized to these membranes to score RFLPs.

\section{Data Analysis}

Year-location combinations were treated as independent environments. Each set of a population within an environment was analyzed as both a lattice and randomized complete block (RCBD) by the ABIYO FORTRAN program (pers. comm., L. L. Darrah). Adjusted means (over replications) for each set at an environment were used where the lattice analysis was more efficient (relative efficiency $>1.04$ ) than the RCBD, while unadjusted means were used when the RCBD was equally efficient. For Populations 1, 2, and 3, means within a set were adjusted to the location mean to remove "set" effects (pers. comm., G.F. Krause, Missouri Agricultural Experiment Station statistician). A combined analysis of variance using means from each environment was conducted to estimate genotype variance, genotype $\times$ environment variance, phenotype variance, and broad-sense heritability on an entry-mean basis. Entry means across environments were used to compute phenotypic correlation coefficients by means of SAS PROC CORR (SAS Institute, Inc., 1998). Standard errors were used to determine whether parental RPR values were significantly different and whether transgressive segregants were present in the population.

MAPMAKER/EXP version 3.0b was used to construct linkage maps (Lander et al., 1987; Lincoln et al., 1992). QTL Cartographer version 1.14d (Basten et al., 1994; Basten et al., 2000) was employed for QTL analysis of family means across environments. Cofactors were identified using forward and backward stepwise regression with $P\left(F_{\text {in }}\right)=P\left(F_{\text {out }}\right)=0.01$, and composite interval mapping (Zeng, 1994) was used to estimate QTL locations and their effects. For each trait-population combination, experiment-wise significance thresholds at $P=0.05$ were determined by analyzing 1000 permutations of the data according to Churchill and Doerge (1994). When multiple peaks were found within a single-marker interval, the location with the highest log-odds ratio (LOD) score was defined as the QTL peak. When multiple peaks were found in adjacent marker intervals, either (i) the location with the largest LOD score was defined as the QTL peak when one peak had a very high LOD score and the other had a relatively low LOD score, (ii) an intermediate location was defined as the QTL peak when the multiple peaks were equal in LOD score and distance from the separating marker, or (iii) each peak was defined as a QTL peak when there was at least a one-half-LOD difference from the peak to the separating marker. A one-LOD drop from the peak position was used as a confidence interval for QTL location. Quantitative trait loci were placed on a composite QTL map on the basis of the markers of Population 3 by means of markers common among the populations. Overlapping confidence intervals were used to determine whether QTL were in common among populations. Estimates of dominance effects as calculated by Zmapqtl in QTL Cartographer (Basten et al., 1994; Basten et al., 2000) were multiplied by two because $\mathrm{F}_{2: 3}$ families were used in the analysis rather than $\mathrm{F}_{2}$ individuals.

The statistical program EPISTACY was used to test for the presence of epistatic interactions between marker pairs at $P<0.001$ (Holland, 1998). To build multilocus models, markers nearest to single-effect QTL, interactions, and the markers involved in the interactions were subjected to stepwise regression at $P<0.05$ by SAS PROC REG (SAS Institute, Inc., 1998). Markers were added to the model in order of increasing $P$-value (forward regression in), and were removed if their significance while in the model exceeded 0.05 (backward regression out).

\section{RESULTS Phenotypic Data Analysis}

Variation for RPR among the $\mathrm{F}_{2: 3}$ families for each of the four populations was highly significant $(P<0.001$, data not shown). The distribution of mean RPR values for $\mathrm{F}_{2: 3}$ families approximated a normal distribution (Fig. 1). Little apparent transgressive segregation was seen in the first three populations; however, a large proportion of the families $(75.0 \%)$ fell outside the range of the parental inbred lines in Population 4 (Fig. 1D). For Populations 1, 2, and 3, the mean RPR values for families and the $F_{1}$ fell within the range of the parental values, whereas in Population 4, both the family mean and the $F_{1}$ RPR values were less than lowest parent, Mo47 (Table 2). Broad-sense heritability estimates for RPR (Table 3) ranged from 0.81 to 0.92 , indicating a strong genetic basis and consistent measurement for RPR.

\section{QTL Analysis}

Empirical threshold LOD values for genome-wise significance at $P<0.05$ as determined by permutation analysis were $3.66,3.53,3.68$, and 3.66 for Populations 1, 2, 3, and 4, respectively. Eight single-effect QTL for RPR were detected in Population 1 (Table 4). Four epistatic interactions were found. Only one of the eight loci involved in the interactions was also detected as a single-effect QTL. A multilocus model including seven single-effect QTL and one interaction accounted for $33.4 \%$ of the phenotypic variation. Six of the eight QTL 

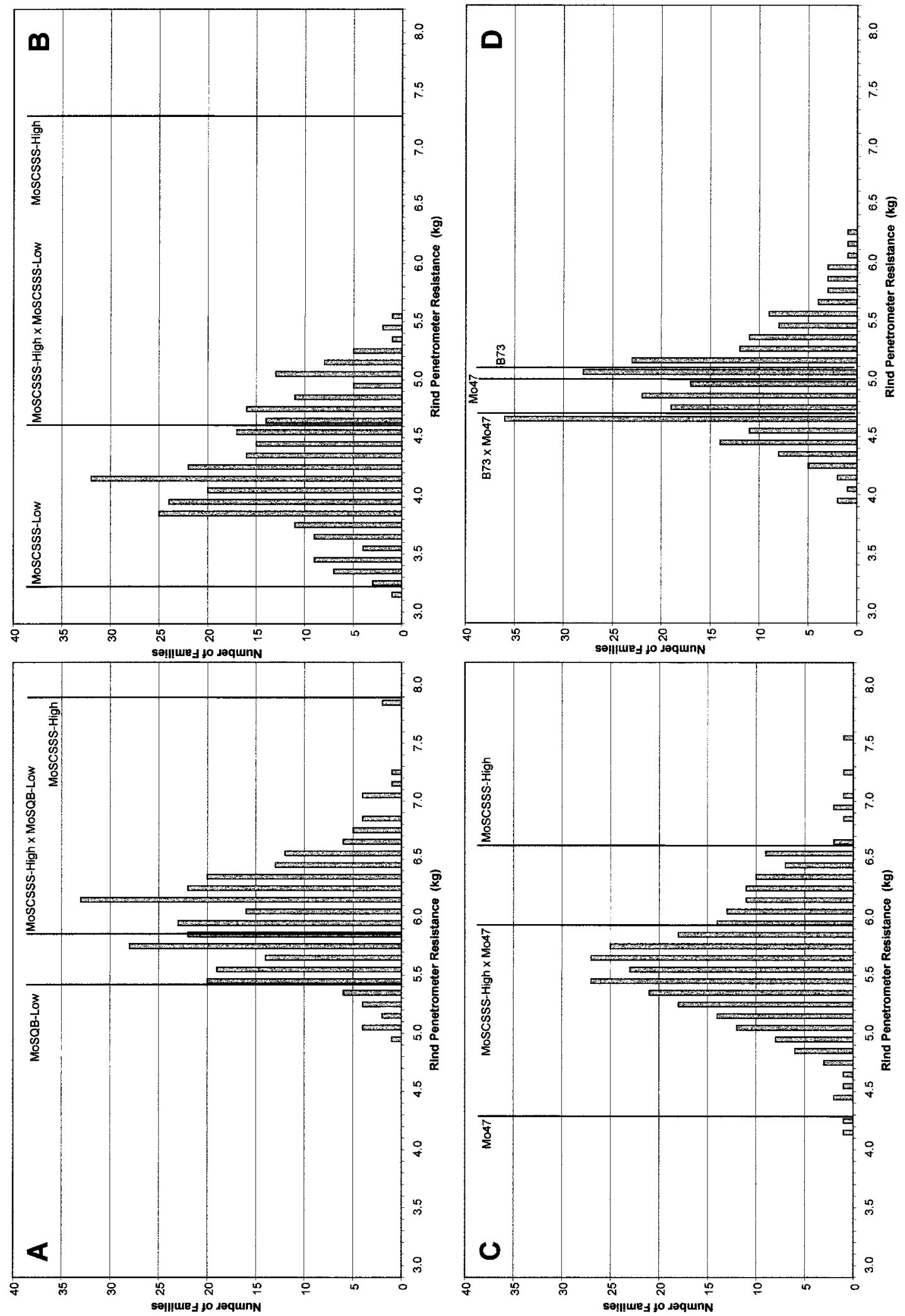

Fig. 1. Frequency distribution of $\mathbf{F}_{2: 3}$ family means for rind penetrometer resistance. (A) Population 1 (MoSCSSS-High1 $\times$ MoSQB-Low) $F_{2: 3}$, (B) Population 2 (MoSCSSS-High2 $\times$ MoSCSSS-Low) $F_{2: 3}$, (C) Population 3 (MoSCSSS-High3 $\times$ Mo47)F $_{2: 3}$, and (D) Population 4 (B73 $\times$ $\mathbf{M o 4 7}_{\mathbf{2}} \mathrm{F}_{2: 3}$. All populations are displayed on a similar scale for comparison purposes. 
Table 2. Means combined over all environments for rind penetrometer resistance in four populations: Population 1, (MoSCSSS-High1 $\times$ MoSQB-Low) $F_{2: 3}$; Population 2, (MoSCSSS-High2 $\times$ MoSCSSS-Low) $_{2: 3}$; Population 3, (MoSCSSS-High3 $\times$ Mo47) $_{2: 3}$; and Population 4, $(\mathbf{M o 4 7} \times \mathbf{B 7 3})_{2: 3 \cdot}$

\begin{tabular}{|c|c|c|c|c|c|}
\hline \multirow{2}{*}{ Population } & \multicolumn{4}{|c|}{$\longrightarrow$ Mean $\longrightarrow$} & $\mathbf{C V}+$ \\
\hline & & - loa & & - & $(\%)$ \\
\hline 1 & $\mathbf{F}_{2: 3}$ families & MoSCSSS-High1 & MoSQB-Low & $\mathbf{F}_{1}$ & 4.6 \\
\hline 2 & $\begin{array}{c}F_{2: 3} \text { families } \\
4.26\end{array}$ & $\begin{array}{c}\text { MoSCSSS-High2 } \\
7.27\end{array}$ & $\begin{array}{c}\text { MoSCSSS-Low } \\
3.24\end{array}$ & $\begin{array}{c}5.00 \\
F_{1} \\
4.63\end{array}$ & 5.6 \\
\hline 3 & $\begin{array}{c}\mathbf{F}_{2: 3} \text { families } \\
\mathbf{5 . 6 6}\end{array}$ & $\begin{array}{c}\text { MoSCSSS-High3 } \\
6.57\end{array}$ & $\begin{array}{r}\text { Mo47 }_{4.31} \\
\end{array}$ & $\begin{array}{c}F_{1} \\
5.93\end{array}$ & \\
\hline 4 & $\begin{array}{c}\mathbf{F}_{2: 3} \text { families } \\
4.95\end{array}$ & $\begin{array}{l}\text { Mo47 } \\
4.98\end{array}$ & $\begin{array}{ll}\text { B73 } & \\
& 5.06\end{array}$ & $\begin{array}{c}\mathbf{F}_{1} \\
\mathbf{4 . 7 1}\end{array}$ & 8. \\
\hline
\end{tabular}

$\dagger$ Coefficient of variation.

alleles that increased RPR originated from MoSQBLow and two originated from MoSCSSS-High1.

Ten single-effect QTL and two epistatic interactions were detected for RPR in Population 2. None of the four loci involved in the interactions were detected as a single-effect QTL. A multilocus model including seven single-effect QTL and one interaction explained $44.8 \%$ of the phenotypic variation. All alleles that increased RPR originated from MoSCSSS-High2.

Eight single-effect QTL for RPR were detected in Population 3. No significant epistatic interactions were detected, and a multilocus model including seven singleeffect QTL accounted for $48.4 \%$ of the phenotypic variation. Six of the eight alleles that increased RPR originated from MoSCSSS-High3, while two alleles originated from Mo47.

Nine single-effect QTL and five epistatic interactions were detected in Population 4. Only two of the 10 loci involved in the interactions were detected as singleeffect QTL. A multilocus model including six singleeffect QTL and two interactions explained $58.7 \%$ of the phenotypic variation. Five of the nine alleles increasing RPR originated from $\mathrm{B} 73$ and four originated from Mo47. The QTL near $u m c 38$ on chromosome $6 \mathrm{~L}$ had a large partial $R^{2}(20.2 \%)$ relative to the other QTL detected in this study.

A composite QTL map based on the linkage map from Population 3 was used to display the relative location of the QTL among the four populations (Fig. 2). Refer to Flint-Garcia et al. (2003, this issue) and Jampatong et al. (2002) for individual linkage maps. Only one region on chromosome 3 contained overlapping confidence intervals from all four populations. Two regions had overlapping QTL from three populations and

Table 3. Variance components and heritability estimates for the analysis of rind penetrometer resistance in four populations: Population 1, (MoSCSSS-High1 $\times$ MoSQB-Low) F $2: 3_{3}$; Population 2, (MoSCSSS-High2 $\times$ MoSCSSS-Low) $F_{2: 3}$; Population 3, $\left(\right.$ MoSCSSS-High3 $\times$ Mo47) $_{2: 3}$; and Population 4, $($ Mo47 $\times$ B73) $\mathbf{F}_{2: 3} \cdot$

\begin{tabular}{ccccc}
\hline & \multicolumn{3}{c}{ Variance components } \\
\cline { 2 - 4 } Population & $\sigma_{\mathrm{g}}^{2}$ & $\sigma_{\mathrm{gxe}}^{2}$ & $\boldsymbol{\sigma}_{\mathrm{ph}}^{2}$ & $\mathbf{H}^{2} \dagger$ \\
\hline 1 & 0.07 & 0.02 & $\mathbf{0 . 0 9}$ & $\mathbf{0 . 8 1}$ \\
2 & $\mathbf{0 . 0 8}$ & $\mathbf{0 . 0 1}$ & $\mathbf{0 . 0 8}$ & $\mathbf{0 . 9 2}$ \\
3 & $\mathbf{0 . 0 8}$ & $\mathbf{0 . 0 1}$ & $\mathbf{0 . 0 9}$ & $\mathbf{0 . 8 8}$ \\
4 & $\mathbf{0 . 1 4}$ & $\mathbf{0 . 0 9}$ & $\mathbf{0 . 1 7}$ & $\mathbf{0 . 8 3}$ \\
\hline
\end{tabular}

$\dagger$ Broad-sense heritability. five regions had overlapping QTL from two populations.

\section{DISCUSSION}

Heritability, the ratio of genotypic variance to phenotypic variance, is a measure of genetic control of a trait. Heritability estimates (Table 3 ) calculated in this study indicated a strong genetic basis for RPR with correspondingly low environmental influence. The ability to accurately and precisely measure RPR over environments was clearly reflected in low CVs (Table 2). Therefore, it should be feasible to identify estimates of genetic effects for QTL for RPR.

Little transgressive segregation was seen in the first three populations; however, a large proportion of the families $(75.0 \%)$ fell outside the range of the parental lines in Population 4 (Fig. 1). This could be explained by the fact that Population 4 was the only population in this study derived from two inbred lines unselected for RPR. The parental lines, B73 and Mo47, may contain complementary QTL for RPR where, through recombination, the wide array of genotypes produced by the $\mathrm{F}_{1}$ result in transgressive segregants in the following generation.

Quantitative traits have been defined as characters that display continuous distribution of phenotypes. The variability is usually associated with the segregation of multiple minor genes, which have small individual effects and are influenced by the environment. The phenotypic distributions shown in Fig. 1 demonstrate the continuous variation of the RPR phenotype in all four populations. The QTL analysis results summarized in Table 4 reveal that RPR is governed by numerous QTL with small-to-moderate effects. Eight, 10, eight, and nine QTL were found in the four populations. Of these QTL, only the QTL on chromosome $6 \mathrm{~L}$ in Population 4 had a partial $R^{2}$ above $15 \%$, indicating a lack of a major QTL for RPR. In addition to a large number of singleeffect QTL, significant epistatic interactions were detected in three of the four populations. Only three of the 22 loci involved in these interactions were detected as single-effect QTL, again indicating the complexity of RPR. The results of the current study are consistent with the results of previous studies investigating the genetic complexity of RPR. Heredia-Diaz et al. (1996) used allelic frequency changes at 16 RFLP loci over 
Table 4. Significant single-effect QTL and epistatic interactions detected for rind penetrometer resistance. Population 1, (MoSCSSSHigh1 $\times$ MoSQB-Low) $F_{2: 3} ;$ Population 2, $\left(\right.$ MoSCSSS-High2 $\times$ MoSCSSS-Low) $F_{2: 3} ;$ Population 3, $\left(\right.$ MoSCSSS-High3 $\times$ Mo47) $_{2: 3} ;$ and Population 4, $\left(\right.$ Mo47 $\times \mathbf{B 7 3}_{)} \mathrm{F}_{2: 3}$.

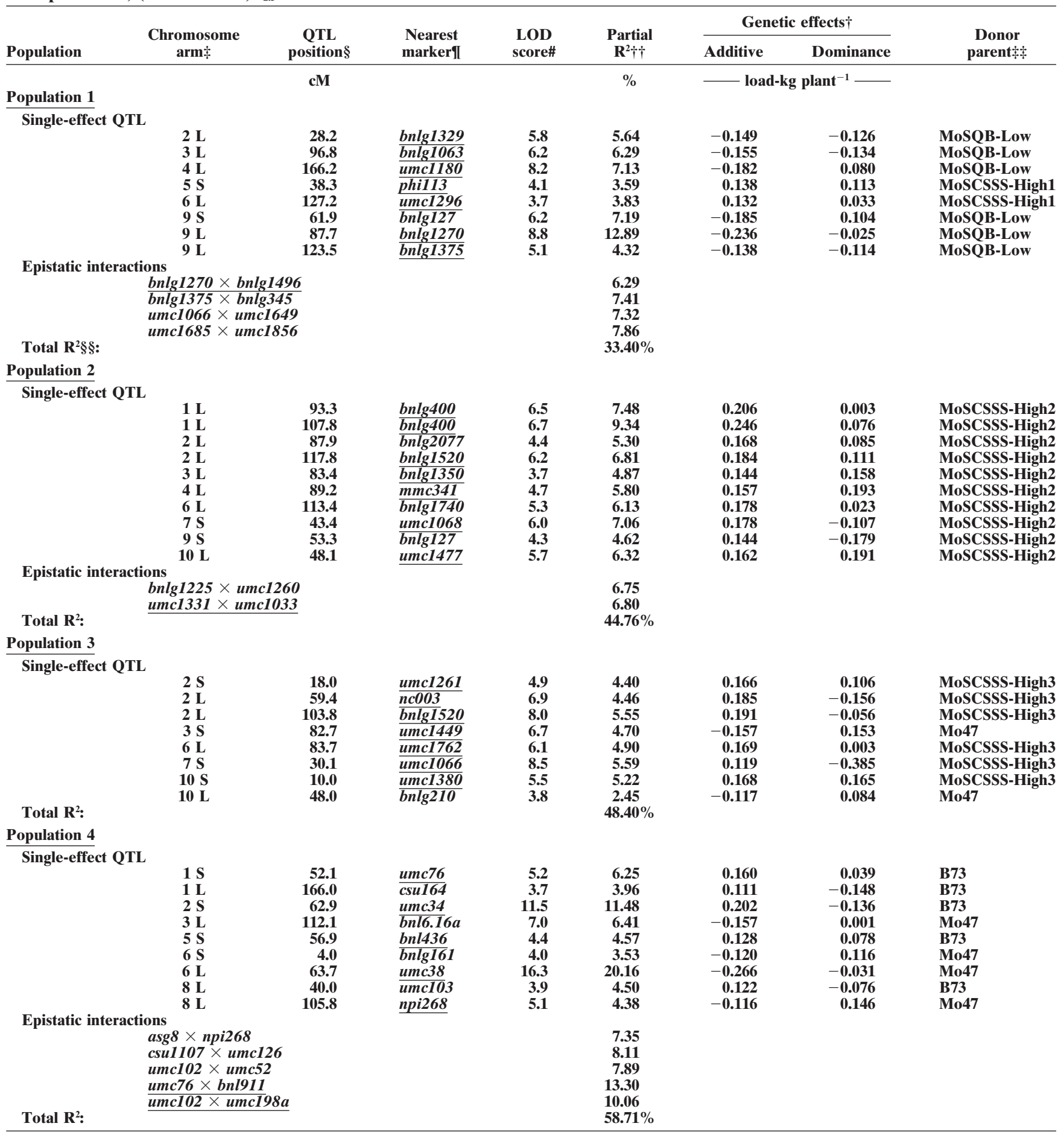

$\dagger$ Dominance effects were multiplied by two since $F_{2: 3}$ families were used in the analysis in place of $F_{2}$ individuals.

S, short arm; L, long arm.

$\$$ Distance measured from the terminal marker on the short arm of the chromosome.

II Underlined single-effect QTL and epistatic interactions were components of the multi-locus model.

\# For the test of $\mathrm{H}_{3}(\mathrm{a} \neq 0, \mathbf{d} \neq 0): \mathrm{H}_{0}(\mathrm{a}=\mathbf{0}, \mathbf{d}=\mathbf{0})$. Likelihood of odds (LOD) scores can be converted to likelihood ratios by multiplying by 4.6052.

$+\dagger$ Percent phenotypic variation explained by the QTL as reported by QTL Cartographer (Basten et al., 2000) for single-effect QTL and by EPISTACY (Holland, 1998) for epistatic interactions.

t+ Donor parent is the parent that contributed the allele responsible for increasing $\mathbf{R P R}$.

$\S \S$ Total phenotypic variation accounted for by a multi-locus model as determined by forward and backward stepwise regression at $P<0.05$. 


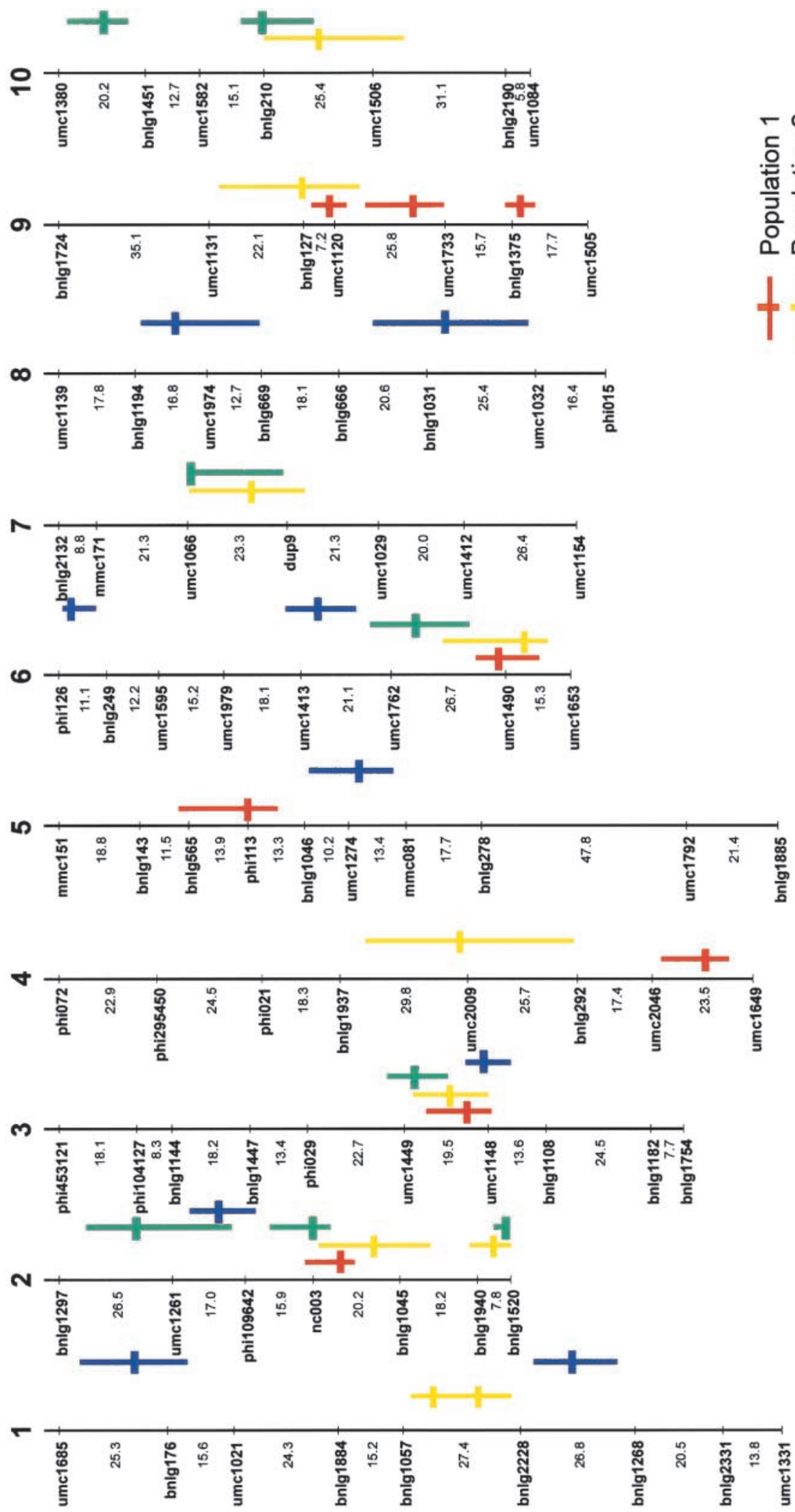

Fig. 2. Relative location of QTL detected by composite interval mapping for rind penetrometer resistance for four populations. Horizontal bars represent QTL peak locations and vertical bars represent a one-LOD confidence interval. Population 1 (MoSCSSS-High1 $\times$ MoSQB-Low) $F_{2: 3}$,

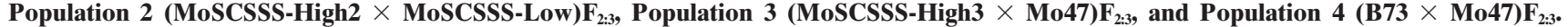
Linkage map is based on Population 3; refer to Flint-Garcia et al. (2003, this issue) and Jampatong et al. (2002) for individual linkage maps. 
seven cycles of selection for RPR and found 12 loci correlated with RPR, three of which accounted for $99 \%$ of the total variation. Lee et al. (1996) found that hyperploidy or hypoploidy for 13 of 18 chromosome arms was associated with significant differences in RPR.

In Population 1, the majority of the alleles that increase RPR originated from MoSQB-low, the low SCS parent. This result was unexpected because the RPR phenotype of the low SCS parent was low. We believe that the correlation between RPR and EH may have contributed to this particular result (Flint-Garcia et al., 2003, this issue). In Population 2, all alleles responsible for increasing RPR originated from the high RPR parent. This result is consistent with bidirectional selection for RPR successfully separating MoSCSSS- $\mathrm{C}_{0}$ into two distinct subpopulations (Alsirt, 1993). Therefore, bidirectional selection was able to partition the high and low RPR alleles into the separate subpopulations. In Population 3, the majority of the high RPR alleles originated from MoSCSSS-High3, with Mo47 contributing only two of the eight favorable alleles. Inbred line Mo47 was developed as a source of resistance to both the firstand second-generation of $\mathrm{ECB}$, and was derived from $50 \%$ exotic germplasm (Barry et al., 1995). Mo47 may contain alleles not normally found in Corn Belt germplasm that increase RPR, but, as expected, the majority of the alleles increasing RPR came from the MoSCSSSHigh3 parent because of its selection for high RPR. In Population 4, five of the nine alleles increasing RPR originated from B73 and four originated from Mo47. This nearly equal contribution of high RPR alleles supports the concept presented earlier that B73 and Mo47 contained complementary sets of alleles at multiple QTL leading to the transgressive segregants for both high and low RPR among the families.

In terms of their QTL profiles, Populations 2 and 3 were most similar, having six QTL in common. Population 1 had only four and two QTL in common with Populations 2 and 3 for two possible reasons. The most likely reason is that most of the high RPR alleles in Population 1 came from the low parent, MoSQB. Because MoSQB was only involved as a parent for Population 1, one would not expect to see the MoSQB alleles in the other three populations. A less likely, but still a plausible reason, is the differences in the degree of heterozygosity between the high RPR parent of the three populations (Table 1). The high RPR parent of Population 1, MoSCSSS-High1, had been self pollinated to produce the high parents of Populations 2 and 3, MoSCSSS-High2 and MoSCSSS-High3. Population 4 was distinctly different than other populations with only two of the 10 QTL in common with any of the other populations. The two QTL in Population 4 were in common with those in Population 3 where Mo47 was the common parent. It is not surprising that Population 4 was different from the other populations since Populations 1, 2, and 3 have a high RPR parent in common. There are various explanations for differences in QTL detected among populations. The first is that QTL analysis will only detect loci that are segregating within the population. If both parents of a population have the same allele at a QTL, that QTL will not be detected in the analysis. The second explanation is that there may be significant epistasis. A QTL in one genetic background may interact with another locus, or a number of loci, to produce a specific genetic effect, whereas in the presence of a different set of background alleles, the QTL may behave another way. A third explanation is that QTL analysis lacks sufficient statistical power to detect QTL with small effects consistently (Beavis, 1998).

The partial $R^{2}$ values on a single-QTL basis were low to moderate, ranging from 2.5 to $12.9 \%$, with the exception of the QTL on chromosome 6 in Population $4\left(R^{2}=20.2 \%\right)$ (Table 4). These data indicate a lack of a major QTL for RPR in contrast to the results from Heredia-Diaz et al. (1996). In the Heredia-Diaz study, allelic frequency changes were surveyed at 16 RFLP loci over six cycles of divergent selection for RPR. We believe that the difference in results of the present study and those of the Heredia-Diaz study lies in the different methodologies and population structures used in the experiments. Many loci underlying RPR may be essential for numerous interconnected biochemical and developmental pathways and, therefore, may show fairly consistent expression among lines, resulting in small QTL effects.

Multilocus models only explained a moderate percent of the total phenotypic variation in the first three populations: $33.4,44.8$, and $48.4 \%$ for Populations 1, 2, and 3 , respectively (Table 4). Because there were no major QTL detected in Populations 1, 2, and 3, the variation in RPR was due exclusively to the segregation of several minor QTL. As discussed, QTL analysis often cannot consistently detect QTL with small effects. In Population 4, however, where there was a QTL with a relatively large effect $\left(R^{2}=20.2 \%\right)$, a larger proportion of the phenotypic variation was explained (58.7\%).

Potential candidate genes for QTL detected in this study include those involved in lignin synthesis, the general phenylpropanoid pathway, and vegetative phase change (Table 5). Magee (1948) reported a wide lignified zone in the rind to be associated with stalk strength. Previous studies involving maize brown-midrib $(\mathrm{bm})$ mutants, showed that $b m 1$ and $b m 3$ caused a significant reduction in lignin content (Muller et al., 1971). Zuber et al. (1977) found a significant decrease in SCS in bm3 mutants compared to their normal counterparts. Several genes encoding other enzymes in the lignin pathway also fall within confidence intervals for QTL detected in this study. Genes that encode enzymes in early steps of the general phenylpropanoid pathway may influence flux through the pathway, and, thus, increase the amount of lignin synthesis. Alternatively, blockage of pathways that compete with the lignin pathway for common substrates may increase lignin production. Mutations at whp1 and $c 2$, block the production of flavanoids and increase production of chlorogenic acid, a phenylpropanoid compound related to lignin precursors (S. Szalma and M. McMullen, unpublished data). Abedon et al. (1999) found that vegetative phase change occurred earlier in populations selected for high RPR than low RPR. Unfortunately, no candidate gene could be identified 
Table 5. Potential candidate genes for rind penetrometer resistance (RPR) QTL.

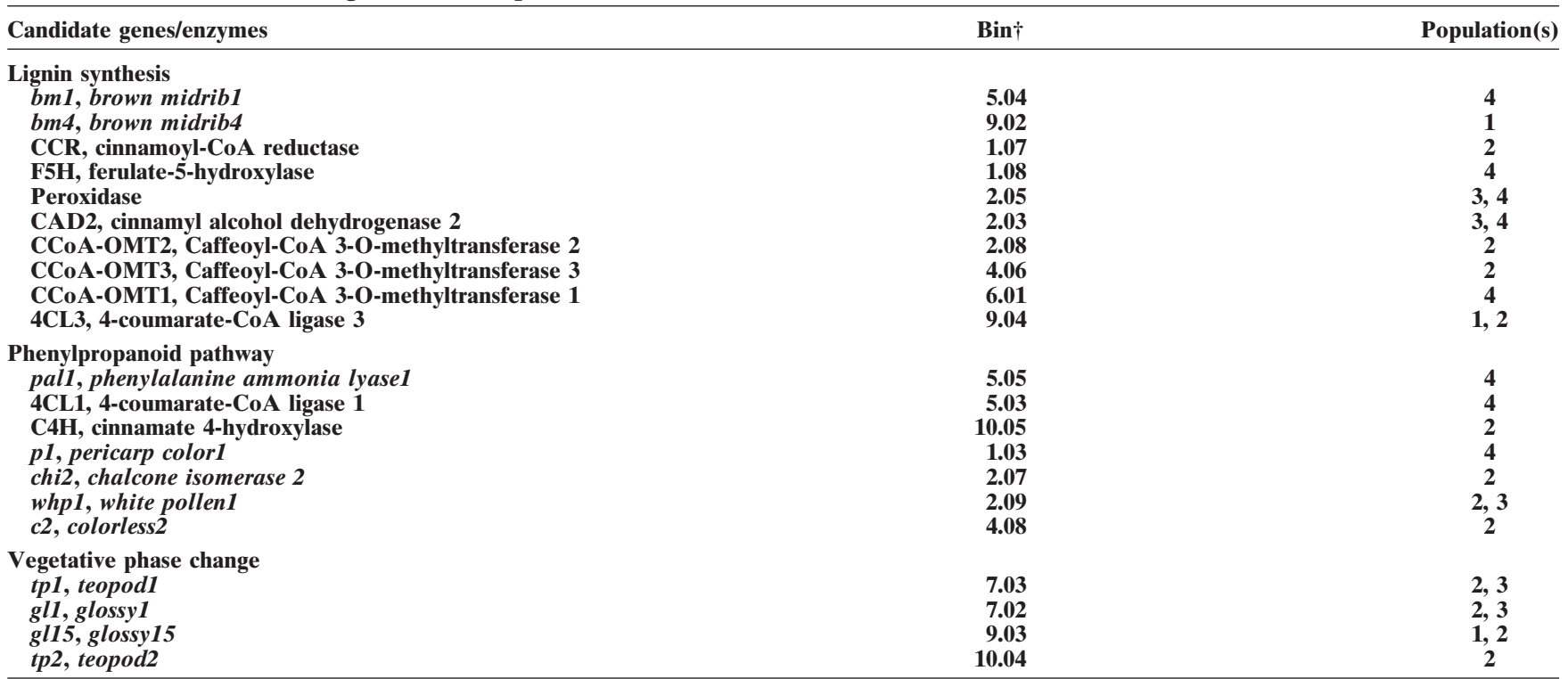

$\dagger$ As reported in the Maize Database, http://www.agron.missouri.edu.

for the QTL on chromosome 3, the only region where there was a QTL in common across all four populations.

Many early stalk lodging studies focused on anatomical and physiological characteristics related to stalk strength and stalk lodging. Rind thickness has been found to be negatively correlated with stalk lodging in several studies (Zuber and Grogan, 1961; Thompson, 1963). Berzonsky et al. (1986) found significant differences for number of vascular bundles, rind-parenchyma interlumen thickness, and percent hypodermal cell wall area among cycles selected for SCS. Magee (1948) reported low bundle number per area of rind, high percentage of sheath per bundle, large stalk diameter, and a wide lignified zone to be associated with stalk strength. Any genes underlying these traits may also be candidate genes for RPR. Unfortunately, little work has been done to investigate the genetic nature of these morphological traits. When the genetics of these traits have been resolved, genes underlying them may coincide with QTL detected in this study.

\section{ACKNOWLEDGMENTS}

The authors would like to acknowledge Julie Barry, Chris Browne, Sansern Jampatong, Sheri Martin, Connie McDevitt, Susan Melia-Hancock, Steve Szalma, and Charlie Thiel for their assistance in phenotypic data collection; Katherine Houchins for screening the parental lines for polymorphic markers; Georgia Davis for making the initial crosses for Population 1 ; and Dr. Gary Krause for statistical guidance. S. Flint-Garcia was supported by the Maize Biology Training Grant, NSF BIR 9420688, and the University of Missouri M.S. Zuber Assistantship Endowment. Research support was provided by USDA-ARS.

\section{REFERENCES}

Abedon, B.G., L.L. Darrah, and W.F. Tracy. 1999. Developmental changes associated with divergent selection for rind penetrometer resistance in the MoSCSSS maize synthetic. Crop Sci. 39:108-114.

Alsirt, A. 1993. Evaluation of frequency distribution changes resulting from bi-directional selection for rind penetrometer resistance in maize. M.Sc. Thesis. Univ. of Missouri, Columbia.

Barry, B.D., A.Q. Antonio, and L.L. Darrah. 1995. Registration of Mo45, Mo46, and Mo47 maize germplasm lines with resistance to European corn borer. Crop Sci. 35:1232-1233.

Basten, C.J., B.S. Weir, and Z.-B. Zeng. 1994. Zmap-a QTL cartographer. p. 65-66. In C. Smith et al. (ed.) Proc. of the 5th World Congress on Genetics Applied to Livestock Production: Computing Strategies and Software. Vol. 22. Organizing Committee, 5th World Congress on Genetics Applied to Livestock Production. Guelph, ON, Canada.

Basten, C.J., B.S. Weir, and Z.-B. Zeng. 2000. QTL Cartographer, Version 1.14. Department of Statistics, North Carolina State University, Raleigh, NC.

Beavis, W.D. 1998. QTL analyses: power, precision, and accuracy. p. 145-162. In A.H. Paterson (ed.) Molecular dissection of complex traits. CRC Press, Boca Raton, FL.

Berzonsky, W.A., J.A. Hawk, and T.Z. Pizzolato. 1986. Anatomical characteristics of three inbred lines and two synthetics recurrently selected for high and low stalk crushing strength. Crop Sci. 26: 482-488.

Chesang-Chumo, J. 1993. Direct and correlated responses to divergent selection for rind penetrometer resistance in MoSCSSS maize synthetic. Ph.D. diss. (Diss. Abstr. Int. 55-04B:1230). Univ. of Missouri, Columbia.

Churchill, G.A., and R.W. Doerge. 1994. Empirical threshold values for quantitative trait mapping. Genetics 138:963-971.

Flint-Garcia, S.A., M.D. McMullen, and L.L. Darrah. 2003. Genetic relationship of stalk strength and ear height in maize. Crop Sci. 43:23-31.

Gerdes, J.T., C.F. Behr, J.G. Coors, and W.F. Tracy. 1993. Compilation of North American maize breeding germplasm. CSSA, Madison, WI.

Heredia-Diaz, O., A. Alsirt, L.L. Darrah, and E.H. Coe. 1996. Allelic frequency changes in the MoSCSSS maize synthetic in response to bi-directional recurrent selection for rind penetrometer resistance. Maydica 41:65-76.

Holland, J.B. 1998. EPISTACY: A SAS program for detecting twolocus epistatic interactions using genetic marker information. J. Hered. 89:374-375.

Jampatong, C., M.D. McMullen, B.D. Barry, L.L. Darrah, P.F. Byrne, and H. Kross. 2002. Quantitative trait loci for first- and secondgeneration European corn borer resistance derived from the maize inbred Mo47. Crop Sci. 42:584-593.

Jampatong, S. 1999. Effect of one- and two-eared selection on stalk strength and other characters in maize. Ph.D. diss. (Diss. Abstr. Int. 60-09B:4314). Univ. of Missouri, Columbia. 
Lander, E., P. Green, J. Abrahamson, A. Barlow, M. Daley, L. Lincoln, and L. Newburg. 1987. MAPMAKER: An interactive computer package for constructing primary genetic linkage maps of experimental and natural populations. Genomics 1:174-181.

Lincoln, S., M. Daly, and E. Lander. 1992. Constructing genetic maps with MAPMAKER/EXP 3.0. Whitehead Institute Technical Report. 3rd edition. Whitehead Institute, Cambridge, MA.

Lee, E.A., L.L. Darrah, and E.H. Coe. 1996. Dosage effects on morphological and quantitative traits in maize aneupliods. Genome 39:898-908.

Magee, J.A. 1948. Histological structure of the stem of Zea mays in relation to stiffness of stalk. Iowa State Coll. J. Sci. 22:257-268.

McDevitt, C.M. 1999. Responses to three cycles of selection with a clamp for plant standability in two maize populations. M.Sc. thesis. Univ. of Missouri, Columbia.

Mihm, J.A. 1985. Breeding for host plant resistance to maize stemborers. Insect Sci. Appl. 6:369-377.

Muller, L.D., R.F. Barnes, L.F. Bauman, and V.F. Colenbrander. 1971. Variations in lignin and other structural components of brown midrib mutants of maize (Zea mays L.). Crop Sci. 11:413-415.

SAS Institute, Inc. 1998. SAS Proprietary Software Version 7. Cary, NC.

Saghai-Maroof, M.A., K.M. Soliman, R. Jogensen, and R.W. Allard. 1984. Ribosomal DNA spacer length in barley: Mendelian inheri- tance, chromosomal location, and population dynamics. Proc. Natl. Acad. Sci. (USA) 81:8014-8018.

Sibale, E.M., L.L. Darrah, and M.S. Zuber. 1992. Comparison of two rind penetrometers for measurement of stalk strength in maize. Maydica 37:111-114.

Spiess, J.E. 1995. Evaluation of selection progress in three dent corn $X$ popcorn populations. M.Sc. thesis. Univ. of Missouri, Columbia.

Smith, D.R., and D.G. White. 1988. Diseases of corn. p. 701-766. In C.F. Sprague and J.W. Dudley (ed.) Corn and corn improvement. 3rd ed. Agron. Monogr.18. ASA, CSSA, and SSSA, Madison, WI.

Thompson, D.L. 1963. Stalk strength of corn as measured by crushing strength and rind thickness. Crop Sci. 3:323-329.

USDA-ARS and Mo. Agric. Exp. Stn. 1986. Release of two dent corn populations. Univ. of Missouri Agric. Exp. Stn., Columbia, MO, and USDA-ARS, Washington, DC.

Zeng, Z.-B. 1994. Precision mapping of quantitative trait loci. Genetics 136:1457-1468.

Zuber, M.S., and C.O. Grogan. 1961. A new technique for measuring stalk strength in corn. Crop Sci. 1:378-380.

Zuber, M.S., T.R. Colbert, and L.F. Bauman. 1977. Effect of brownmidrib-3 mutant in maize (Zea mays L.) on stalk strength. Z Pflanzenzüchtg. 79:310-314.

Zuber, M.S., T.R. Colbert, and L.L. Darrah. 1980. Effect of recurrent selection for crushing strength on several stalk components in maize. Crop Sci. 20:711-717. 